\title{
Optimized imaging of the lower abdomen and pelvic region in hepatocyte-specific MRI: evaluation of a whole-abdomen first-pass shuttle protocol in patients with neuroendocrine neoplasms
}

Acta Radiologica

2019, Vol. 60(9) 1074-1083

(C) The Foundation Acta Radiologica 2018

Article reuse guidelines: sagepub.com/journals-permissions DOI: $10.1177 / 0284185118817936$ journals.sagepub.com/home/acr

@SAGE

\author{
Uli Fehrenbach' ${ }^{\mathbb{D}}$, Johannes Kahn', Ute Fahlenkamp' $\mathbb{D}$, \\ Alexander Baur ${ }^{1,2}$, Marianne Pavel ${ }^{3,4}$, Dominik Geisel' and \\ Timm Denecke ${ }^{\mathrm{l}, 2}$
}

\begin{abstract}
Background: Gadoxetic acid (Gd-EOB) has shown its advantages in hepatic tumor evaluation besides its disadvantages in extrahepatic staging due to its short hemodynamic interval. This results in the dilemma of which contrast agent to choose for whole abdominal magnetic resonance imaging (MRI).

Purpose: To evaluate the achievable contrast of a shuttle protocol for Gd-EOB MRI with accelerated 3D-TI-weighted sequences enabling complete dynamic liver imaging plus first-pass pelvic imaging.

Material and Methods: Seventy-four patients with abdominal neuroendocrine neoplasms were scanned with a protocol including a first-pass pelvic (pelvic $\mathrm{FP}_{\text {) }}$ sequence in Gd-EOB MRI. Acquisition of this pelvic $\mathrm{FP}_{\text {sequence was }}$ between portal venous and venous liver phase. Pelvic vessel and tissue enhancement was compared to a commonly acquired late/transitional phase sequence. In the same patients, liver enhancement was compared to a standard Gd-EOB protocol $(n=55)$ as well as to vessel contrast in previously acquired MRI with extracellular contrast medium $(\mathrm{ECCM})(\mathrm{n}=14)$.

Results: Pelvic vessel and lymph node enhancement showed significantly higher signal intensities (SI) in pelvic ${ }_{F P}$ than in late phase sequences with Gd-EOB $(P<0.001)$. Liver enhancement showed no significant differences compared to the

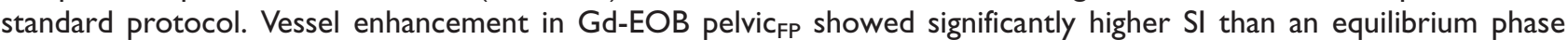
sequence with $\operatorname{ECCM}(P=0.003-0.04)$.

Conclusion: Including a pelvic $\mathrm{FP}_{\mathrm{FP}}$ sequence between portal venous and venous liver phases is technically feasible and achieves improved contrast in the pelvis in Gd-EOB MRI. There was no quality loss in liver enhancement compared to a regular Gd-EOB liver examination. Gd-EOB pelvic $\mathrm{FP}_{\mathrm{FP}}$ vessel enhancement was not inferior to an equilibrium phase with ECCM.
\end{abstract}

\section{Keywords}

Abdomen/Gl, magnetic resonance imaging, liver, pelvis, staging, technical aspects

Date received: 16 August 2018; accepted: II November 2018

\footnotetext{
'Klinik für Radiologie, Charité - Universitätsmedizin Berlin, Berlin, Germany

${ }^{2}$ Bereich klinische Nuklearmedizin, Charité - Universitätsmedizin Berlin, Berlin, Germany

${ }^{3}$ Medizinische Klinik m.S. Hepatologie und Gastroenterologie, Charité Universitätsmedizin Berlin, Berlin, Germany
}

${ }^{4}$ Medizinische Klinik, Endokrinologie, Universitätsklinikum Erlangen, Erlangen, Germany

Corresponding author:

Timm Denecke, Klinik für Radiologie, Campus Virchow-Klinikum, Charité - Universitätsmedizin Berlin, Augustenburger Platz I, I3353 Berlin, Germany.

Email: timm.denecke@charite.de 


\section{Introduction}

Staging of many cancers - especially of gastrointestinal (GI) origin - has to comprise optimal liver imaging as this is the most common site of metastases and frequent site of abdominal primary tumors. Contrast-enhanced magnetic resonance imaging (MRI) is suitable and being increasingly used for this indication $(1,2)$.

For dedicated liver MRI, hepatocyte-specific contrast agents, especially gadoxetic acid (Gd-EOB), with the most intensive hepatic properties, became popular in recent years because of their better detection and characterization of liver lesions due to the additional hepatobiliary phase (3-7). However, Gd-EOB is a linear Gd-compound and, as such, is regarded to be less stable. The use of Gd-EOB might lead to tissue deposition of $\mathrm{Gd}$ and its use is restricted to dedicated hepatic imaging only $(8,9)$. Furthermore, Gd-EOBenhanced MRI shows deficiencies in extrahepatic tumor staging because of inferior vascular and tissue enhancement and rapid decay of T1-shortening effects in the vascular pool compared to extracellular contrast media (ECCM) because of the lower amount of gadolinium per dose and the more rapid elimination from the blood pool (10-12).

In fact, Gd-EOB achieves relatively good tissue contrasts in the extrahepatic upper abdomen during the first-pass contrast phases of dynamic liver acquisition (10). However, if the lower abdomen is intended to be included to perform a whole abdominal staging, a subsequent scan of the lower abdomen and pelvic region during the same examination would be necessary. Such a late phase scan in the transitional phase of Gd-EOB lacks tissue and vascular contrast enhancement compared to ECCM-enhanced MRI.

However, scanning of the lower abdomen is crucial to detect primary tumors, lymph node metastases, peritoneal spread, and skeletal lesions, leaving the radiologist with the dilemma of which contrast medium to choose for whole abdominal imaging: should the focus of the examination be on optimal liver imaging or on extrahepatic lower abdominal imaging. Therefore, an MR examination technique would be desirable enabling whole abdominal imaging with Gd-EOB without impairment of the hepatic image quality along with tissue and vessel enhancement in the lower abdomen and pelvis not inferior to that achieved with a whole abdominal ECCM-enhanced MRI. This would make trade-off or additional examinations and contrast applications no longer necessary. A promising approach to this is to extend the contrastenhanced imaging during the dynamic phases from the upper abdomen and liver to the lower abdomen and pelvis. This becomes possible with modern accelerated three-dimensional (3D) T1-weighted (T1W) volume interpolated breath-hold examination (VIBE) sequences such as CAIPIRINHA (Controlled Aliasing in Parallel Imaging Results in Higher Acceleration) combined with rapid table shuttling.

The aim of this study was to analyze the achievable tissue and vessel contrast enhancement in the lower abdomen and the liver using a whole abdomen and pelvis MRI with Gd-EOB comprising first-pass contrast-enhanced lower abdomen and pelvic imaging between portal venous and venous liver phase. Pelvic enhancement was compared with pelvic Gd-EOB enhanced imaging after the dynamic liver acquisition and also whole abdominal MRI with ECCM.

\section{Material and Methods}

\section{Study design/inclusion criteria}

The study was designed as a retrospective cohort study with inter- and intra-individual evaluation. The study was approved by the institutional review board and informed consent was waived.

A database search was performed to find patients examined at our institution with abdominal MRI for neuroendocrine neoplasms and abdominal tumor burden who had received liver-specific MRI examinations as follow-up scans during the one-year study period from June 2015 to June 2016. Further inclusion criteria were the employment of the first-pass pelvic protocol and completion of all sequences to be evaluated in this study. Exclusion criteria were insufficient or incomplete acquisition of MRI sequences. All eligible patients of the enrollment period completed the whole MR scan, so no patient had to be excluded.

Gd-EOB MRI scans with the standard protocol (without first-pass pelvic sequence) of the same patients were used as a reference. Previously acquired abdominal MRI scans with gadobutrol as ECCM were also evaluated.

\section{Magnetic resonance imaging}

MRI was obtained with 1.5-T or 3-T systems using the same phased-array body coils in all patients (MAGNETOM Aera/Skyra, Siemens Healthcare, Erlangen, Germany). All Gd-EOB examination protocols comprised T1W images in opposed- and in-phase pre-contrast as well as a 3D T1W GRE sequence with fat saturation (FS). After intravenous application of Gd-EOB (Primovist, Bayer, Berlin, Germany; $0.025 \mathrm{mmol} / \mathrm{kg}$ body weight; automatic injection at $2 \mathrm{~mL} / \mathrm{s}$ flow rate, $40 \mathrm{~mL}$ saline flush) dynamic 3D T1W FS GRE sequences (VIBE, CAIPIRINHA) were acquired using breath-hold technique. T2weighted (T2W) sequences, with and without FS, and 
diffusion-weighted imaging (DWI) sequences were acquired to bridge the interval to hepatobiliary plateau phase. T1W imaging (VIBE, Flash, and STARVIBE) was repeated 20-25 min after contrast administration in the hepatobiliary plateau phase.

In the standard protocol, the multi-phase contrast sequences, 3D T1W FS GRE, start with an arterial phase sequence of the liver with a fixed delay of $15 \mathrm{~s}$ after contrast injection. Subsequently, portal venous ( $\sim 53 \mathrm{~s}$ delay), venous ( $\sim 98 \mathrm{~s}$ delay), and transitional ( $\sim 144 \mathrm{~s}$ delay) phase sequences of the liver were acquired. After acquisition of the multi-phase liver sequences acquisition of a late pelvic (pelvic LATE$_{\text {) }}$ sequence $(\sim 186 \mathrm{~s}$ delay) started in a transitional contrast phase.

In the evaluated first-pass pelvic protocol (FP protocol), the arterial phase sequence of the liver also started with a fixed delay of $15 \mathrm{~s}$ after contrast injection followed by a portal venous liver phase ( $\sim 56$ s delay). Between the portal venous and venous liver phase sequences, acquisition of the pelvic first-pass (pelvic ${ }_{\mathrm{FP}}$ ) sequence started by shuttling the patients table to the lower abdomen $(\sim 124 \mathrm{~s}$ delay). The pelvic $_{\mathrm{FP}}$ sequence was followed by two more liver sequences (venous and transitional phases) by shuttling the patient table back to the upper abdominal scan field ( $\sim 152$ and $181 \mathrm{~s}$ delay) (Fig. 1). For evaluation purposes, another 3D T1W 3D FS GRE sequence of the pelvic region followed as a pelvic LATE $_{\text {sequence resem- }}$ bling the pelvic LATE $_{\text {sequence of the standard protocol }}$ ( 217 s delay).

In 14 of our patients an additional, previously acquired MRI with ECCM was available for evaluation (at 1.5-T field strength). In these scans, gadobutrol (Gadovist, Bayer, Berlin, Germany) was used as the contrast agent $(0.1 \mathrm{~mL} / \mathrm{kg}$ body weight). In the ECCM protocol, a pelvic sequence was acquired after multi-phase liver imaging in a pelvic $_{\text {LATE }}$ equilibrium phase (at approximately $185 \mathrm{~s}$ delay).

\section{Quantitative assessment and statistical analysis}

All available pelvic ${ }_{\mathrm{FP}}$ and pelvic $_{\mathrm{LATE}}$ were analyzed. Vessel enhancement was measured with circular regions of interest (ROIs) in the iliac veins, inferior vena cava (IVC), and abdominal aorta (standardized ROI diameter of $5 \mathrm{~mm}$ ). Tissue enhancement was obtained by circular ROIs in retroperitoneal lymph nodes (fat in the hilar region was excluded when reasonably possible) and lower back muscles (erector spinae muscles). Liver enhancement was measured in venous phase liver sequences with circular ROIs (standardized diameter of $5 \mathrm{~mm}$ in vessels and $10 \mathrm{~mm}$ in parenchyma) in central liver parenchyma (segment 7), portal vein (central), and hepatic veins (central, near IVC). All ROIs were placed by an experienced abdominal radiologist (4+ years of experience).

For quantitative analysis, relative signal intensities (SI) after contrast administration were calculated by the formula: ((SI $\left.\left.\mathrm{SI}_{\text {post-contrast }}-\mathrm{SI}_{\text {pre-contrast }}\right) / \mathrm{SI}_{\text {pre-contrast }}\right) * 100 \%$.

Intra-individual comparison of pelvic vessel enhancement between ECCM-MRI and Gd-EOB MRI was also performed as secondary findings. In these additional cases $(n=14)$, relative SI of vessel enhancement had to be calculated in comparison to lower back muscle enhancement because of a missing pre-contrast T1W sequence in the ECCM protocol. Therefore, relative SI had to be calculated by the formula: (( $\left.\left.\mathrm{SI}_{\mathrm{ROI}}-\mathrm{SI}_{\text {muscle }}\right) / \mathrm{SI}_{\text {muscle }}\right) * 100 \%$.

SPSS (IBM, Version 21) was used for statistical analysis. Non-normal distribution of the data was confirmed by Kolmogorov-Smirnov test. Relative signal intensities were analyzed by non-parametric Wilcoxon

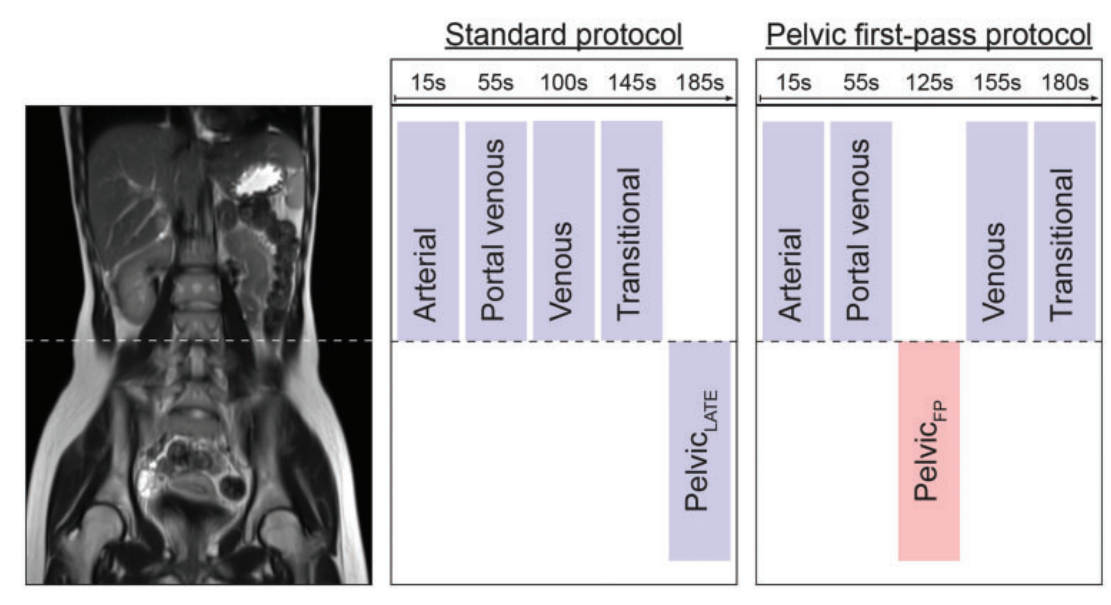

Fig. I. Proposal of sequence acquisition in the FP protocol in comparison to a standard protocol in Gd-EOB MRI. For evaluation purposes, the FP protocol also contained a pelvic LATE sequence during the study period. 
and Mann-Whitney U tests. $P$ values $<0.05$ were considered significant for all statistical analyses.

\section{Results}

\section{Patient characteristics}

A total of 74 patients with abdominal neuroendocrine neoplasms were found eligible and were enrolled. The most common primary tumor sites were the ileum/jejunum (28 patients) and pancreas (26 patients). Other locations of the primary tumor sites were the large bowel (four patients), duodenum (three patients), lung (four patients), and appendix, stomach, breast, and uterus (one patient each). In five patients, the tumor was classified as carcinoma of unknown primary (CUP) (Table 1).

In these patients, $82(1.5 \mathrm{~T}=56 ; 3 \mathrm{~T}=26) \mathrm{MRI}$ scans with the first-pass protocol were performed. The eight repeated FP exams in the patients' collective were regarded as independent. Fifty-five of these patients $(1.5 \mathrm{~T}=38 ; 3 \mathrm{~T}=17)$ also received the standard protocol without the pelvic ${ }_{\mathrm{FP}}$ sequence. The mean time in between the FP and the standard protocol in these patients was 191 days (range $=82-383$ days). Additionally, 14 of the patients previously received an MRI examination $(1.5 \mathrm{~T})$ of the whole abdomen with gadobutrol as ECCM and the abovementioned ECCM protocol. The mean time between the FP and the ECCM protocol in these patients was 242 days (range $=101-572$ days) $($ Table 1$)$.

\section{Delay of the acquired sequences}

In the FP protocol, the pelvic ${ }_{\mathrm{FP}}$ sequence started approximately $124 \mathrm{~s}$ after contrast injection. As a result of the additional sequence, the venous phase liver sequence started with a delay of $152 \mathrm{~s}$ in the FP protocol and after approximately $98 \mathrm{~s}$ in the standard protocol. Therefore, in the FP protocol, the venous phase liver sequence was delayed for about $54 \mathrm{~s}$ on average in comparison to the standard protocol. The transitional phase sequence of the liver started with a delay of approximately $181 \mathrm{~s}$ in the FP protocol and $144 \mathrm{~s}$ in the standard protocol. The pelvic LATE sequence in the FP protocol had a delay of $217 \mathrm{~s}$ and $186 \mathrm{~s}$ in the standard protocol (Table 2).

\section{Lower abdomen and pelvic region}

In the FP protocol, the pelvic LATE $_{\text {sequence was }}$ delayed for about $30 \mathrm{~s}$ in comparison to the standard protocol without the pelvic ${ }_{\mathrm{FP}}$ sequence $($ delay $=217 \mathrm{~s}$ vs. $186 \mathrm{~s}$ ). By comparing the pelvic LATE $_{\text {s sequences }}$ from the FP $(\mathrm{n}=82)$ and standard $(\mathrm{n}=55)$ protocol, there was no statistically significant difference in
Table I. Patients' characteristics.

\begin{tabular}{lll}
\hline Characteristics & Field strength (T) & $\mathrm{n}$ \\
\hline Average age (years (range)) & 62.8 (30-85) \\
Gender & \\
Female & 44 \\
Male & 30 \\
Primary tumor site & \\
Ileum/Jejunum & 28 \\
Pancreas & 26 \\
Duodenum & 3 \\
Large bowel & 4 \\
Lung & 4 \\
CUP & & 5 \\
Others & 4 \\
MRI protocol & \\
FP protocol & 1.5 & 56 \\
& 3 & 26 \\
Standard protocol & 1.5 & 38 \\
& 3 & 17 \\
ECCM protocol & 1.5 & 14 \\
\hline
\end{tabular}

CUP, cancer of unknown primary; FP, first-pass; ECCM, extracellular contrast material.

Table 2. Acquisition delays of FP protocol and standard protocol.

\begin{tabular}{lccccc}
\hline & \multicolumn{2}{c}{ FP protocol } & & \multicolumn{2}{c}{ Standard protocol } \\
\cline { 2 - 3 } Sequence & Delay (s) & SD & & Delay (s) & SD \\
\hline Arterial liver & 15 & - & & 15 & - \\
Portal venous liver & 56 & 7 & & 53 & 9 \\
Pelvic & 124 & 24 & & - & - \\
Venous liver & 152 & 25 & 98 & 19 \\
Transitional liver & 181 & 25 & & 144 & 24 \\
Pelvic & 217 & 24 & & 186 & 25 \\
\hline
\end{tabular}

FP, first-pass; SI, signal intensities; SD, standard deviation.

relative SI of vessels and tissue at both field strengths. Therefore, pelvic LATE $_{\text {f }}$ of the FP protocol can be seen as representative despite the delay compared to the standard protocol (Table 3).

Intra-individual (paired) comparison of pelvic ${ }_{\mathrm{FP}}$ and pelvic $_{\text {LATE }}$ in the FP protocol showed statistically significant improvement of relative SI of the pelvic ${ }_{\mathrm{FP}}$ sequence in all measured regions at $1.5 \mathrm{~T}$ and $3 \mathrm{~T}$ : iliac veins, inferior vena cava, aorta, and lymph nodes $(P<0.001)$ (Table 3 and Fig. 2).

\section{Dynamic liver acquisition}

Groupwise comparison of liver enhancement (venous phase liver sequences) between the FP protocol $(\mathrm{n}=82)$ and the standard protocol $(\mathrm{n}=55)$ showed no significant differences in enhancement of liver parenchyma 
Table 3. Comparison of pelvic $\mathrm{LATE}_{\mathrm{LAT}}$ sequences in FP protocol and standard protocol and intra-individual comparison of pelvic $\mathrm{FP}_{\text {and }}$

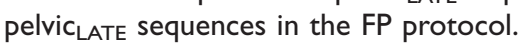

\begin{tabular}{|c|c|c|c|c|c|c|c|c|}
\hline & \multirow[b]{3}{*}{ Field strength $(\mathrm{T})$} & \multicolumn{3}{|c|}{ FP protocol } & \multicolumn{3}{|c|}{ Standard protocol } & \multirow{3}{*}{$\frac{\text { Significance }}{P \text { value }}$} \\
\hline & & \multicolumn{3}{|c|}{ Pelvic $_{\text {LATE }}(n=82)$} & \multicolumn{3}{|c|}{ Pelvic $_{\text {LATE }}(n=55)$} & \\
\hline & & $\mathrm{n}$ & Relative SI & SD & $\mathrm{n}$ & Relative SI & SD & \\
\hline \multirow[t]{2}{*}{ Iliac veins } & 1.5 & 56 & 65.58 & 36.70 & 38 & 74.42 & 36.40 & 0.22 \\
\hline & 3 & 26 & 68.74 & 32.29 & 17 & 75.18 & 30.13 & 0.30 \\
\hline \multirow[t]{2}{*}{ IVC } & 1.5 & 56 & 51.32 & 31.07 & 38 & 52.09 & 35.92 & 0.97 \\
\hline & 3 & 26 & 57.98 & 34.83 & 17 & 70.10 & 30.84 & 0.15 \\
\hline \multirow[t]{2}{*}{ Aorta } & 1.5 & 56 & 113.70 & 53.19 & 38 & $1 \mid 4.88$ & 57.65 & 0.86 \\
\hline & 3 & 26 & 135.54 & 82.54 & 17 & 119.79 & 34.11 & 0.88 \\
\hline \multirow[t]{5}{*}{ Lymph nodes } & 1.5 & 56 & 33.24 & 26.32 & 38 & 35.54 & 23.71 & 0.47 \\
\hline & 3 & 26 & 62.85 & 55.78 & 17 & 60.00 & 33.50 & 0.88 \\
\hline & & \multicolumn{3}{|c|}{ FP protocol } & \multicolumn{3}{|c|}{ FP protocol } & \\
\hline & & \multicolumn{3}{|c|}{ Pelvic $_{\mathrm{FP}}(\mathrm{n}=82)$} & \multicolumn{3}{|c|}{ Pelvic $_{\text {LATE }}(n=82)$} & Significance \\
\hline & Field strength $(\mathrm{T})$ & $\mathrm{n}$ & Relative SI & SD & $\mathrm{n}$ & Relative SI & SD & $P$ value \\
\hline \multirow[t]{2}{*}{ Iliac veins } & 1.5 & 56 & 79.59 & 45.27 & 56 & 65.58 & 36.70 & $<0.001$ \\
\hline & 3 & 26 & 109.37 & 37.34 & 26 & 68.74 & 32.29 & $<0.001$ \\
\hline \multirow[t]{2}{*}{ IVC } & 1.5 & 56 & 67.83 & 38.50 & 56 & 51.32 & 31.07 & $<0.001$ \\
\hline & 3 & 26 & 87.82 & 39.21 & 26 & 57.98 & 34.83 & $<0.001$ \\
\hline \multirow[t]{2}{*}{ Aorta } & 1.5 & 56 & $128.9 \mid$ & 45.62 & 56 & 113.70 & 53.19 & $<0.001$ \\
\hline & 3 & 26 & 170.15 & 49.14 & 26 & 135.54 & 82.54 & $<0.001$ \\
\hline \multirow[t]{2}{*}{ Lymph nodes } & 1.5 & 56 & 49.64 & 24.53 & 56 & 33.24 & 26.32 & $<0.001$ \\
\hline & 3 & 26 & 78.74 & 49.43 & 26 & 62.85 & 55.78 & $<0.001$ \\
\hline
\end{tabular}

IVC, inferior vena cava; FP, first-pass; SI, signal intensity; SD, standard deviation.

and hepatic veins at $1.5 \mathrm{~T}$ and $3 \mathrm{~T}$. The portal vein showed significantly higher relative SI in the standard protocol at $1.5 \mathrm{~T}(P<0.001)$. Portal vein SI also showed a statistical trend to higher SI in the standard protocol at $3 \mathrm{~T}(P=0.06)$ (Table 4$)$.

\section{Comparison with ECCM}

Intra-individual (paired) comparison of pelvic ${ }_{\mathrm{FP}}$ sequence of the FP protocol $(n=14)$ to pelvic ${ }_{\text {LATE }}$ sequence of previously acquired ECCM MRI scans with the ECCM protocol $(n=14)$ showed a significantly higher relative SI of pelvic vessels (iliac veins, IVC, and aorta) in the FP protocol $(P=0.003-0.04)$ at $1.5 \mathrm{~T}$ (Table 5).

\section{Discussion}

In order to enable whole abdominal imaging via contrast-enhanced MRI combined with the full diagnostic advantage of hepatic staging with Gd-EOB as the most hepatocyte specific contrast agent available on the market in a single examination, a novel scan protocol was analyzed in the present study. Taking advantage of technical developments regarding MR hardware and sequence technology allowing rapid 3D T1W breath-hold scanning, a FP vascular phase scan of the lower abdomen and pelvis (pelvic ${ }_{\mathrm{FP}}$ ) was inserted in the dynamic scan phases acquired from the upper abdomen at the level of the liver between the portal venous and venous phase. This scan protocol was analyzed in a retrospective approach in 74 patients with neuroendocrine tumors scheduled for abdominal restaging at $1.5 \mathrm{~T}$ and $3 \mathrm{~T}$ MRI field strengths. The study compared the Gd-EOB enhanced pelvic $_{F P}$ sequence with a commonly acquired late sequence of the lower abdomen and pelvis after the dynamic liver phases (pelvic $c_{\mathrm{LATE}}$ ). The pelvic $\mathrm{FP}_{\mathrm{F}}$ sequence was also compared to an equilibrium pelvic sequence acquired in a conventional scan protocol using ECCM. The results showed improved vessel and tissue enhancement of the pelvic $\mathrm{FP}_{\mathrm{FP}}$ sequence com-

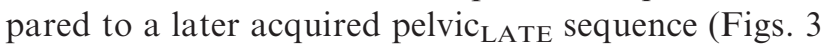
and 4). The vessel enhancement of Gd-EOB in this early pelvic $\mathrm{FP}_{\mathrm{FP}}$ sequence was also higher than in an equilibrium phase ECCM MRI examination of the lower abdomen. There was no relevant trade off in the contrast characteristic of the liver on the venous and transitional phase images caused by the insertion of the pelvic FP $_{\text {sequence. }}$ 

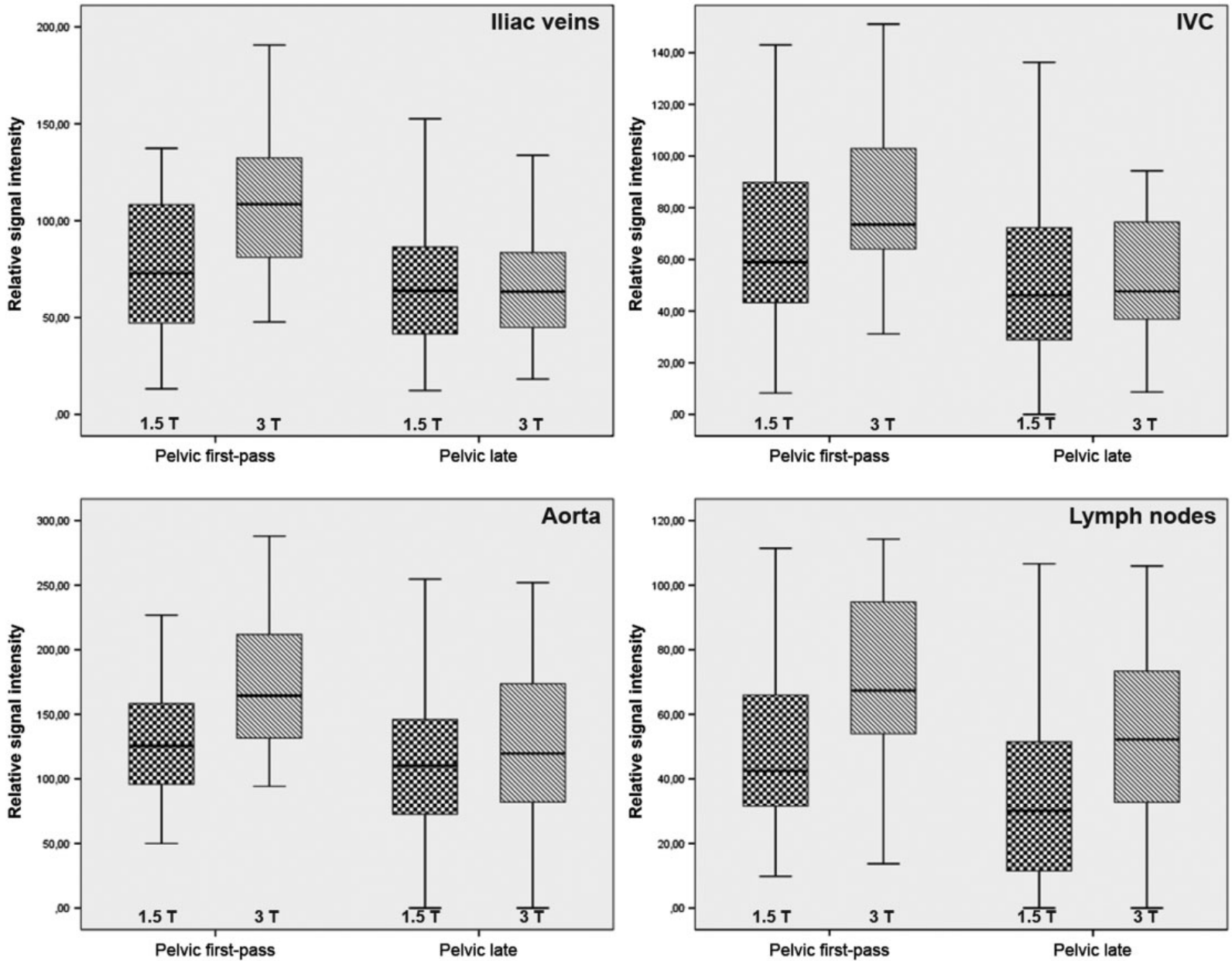

Fig. 2. Boxplots comparing pelvic FP $_{\text {a }}$ and pelvic $\mathrm{LATE}_{\mathrm{L}}$ in $\mathrm{FP}$ protocol at $\mathrm{I} .5 \mathrm{~T}$ and $3 \mathrm{~T}(P<0.00 \mathrm{I})$.

Table 4. Comparison of hepatic enhancement between FP protocol and standard protocol in venous phase liver sequence.

\begin{tabular}{|c|c|c|c|c|c|c|c|c|}
\hline & \multirow[b]{3}{*}{ Field strength $(\mathrm{T})$} & \multirow[b]{3}{*}{$\mathrm{n}$} & \multicolumn{2}{|l|}{ FP protocol } & \multirow[b]{3}{*}{$\mathrm{n}$} & \multicolumn{2}{|c|}{ Standard protocol } & \multirow[b]{2}{*}{ Significance } \\
\hline & & & \multicolumn{2}{|c|}{ Venous liver $(n=82)$} & & \multicolumn{2}{|c|}{ Venous liver $(n=55)$} & \\
\hline & & & Relative SI & SD & & Relative SI & SD & $P$ value \\
\hline \multirow[t]{2}{*}{ Parenchyma } & 1.5 & 56 & 51.17 & 10.54 & 38 & 53.00 & 10.15 & 0.31 \\
\hline & 3 & 26 & 70.94 & 21.52 & 17 & 72.11 & 22.34 & 0.98 \\
\hline \multirow{2}{*}{ Portal vein } & 1.5 & 56 & 155.75 & 34.67 & 38 & 200.88 & 48.50 & $<0.001$ \\
\hline & 3 & 26 & 180.40 & 43.72 & 17 & 201.59 & 31.03 & 0.06 \\
\hline \multirow[t]{2}{*}{ Hepatic veins } & 1.5 & 56 & 157.97 & 52.96 & 38 & 174.08 & 109.76 & 0.32 \\
\hline & 3 & 26 & 166.65 & 42.06 & 17 & 172.11 & 55.02 & 0.64 \\
\hline
\end{tabular}

FP, first-pass; SI, signal intensity; SD, standard deviation.

The idea of extending MR diagnostics with Gd-EOB from the liver to the entire abdomen arises from the need for whole abdominal staging of GI malignancies without diagnostic trade off or additional examinations with extra Gd doses $(1,13)$. The prerequisite of such an examination protocol would be having the full diagnostic power inside the liver along with sufficient diagnostic quality in the extrahepatic region. Gd-EOB, with just one-quarter amount of $\mathrm{Gd}$ per administration partially compensated for by a greater T1-relaxivity due to a higher rate of plasma protein binding (11) has a lesser total T1-shortening effect than ECCM and Gd-BOPTA 


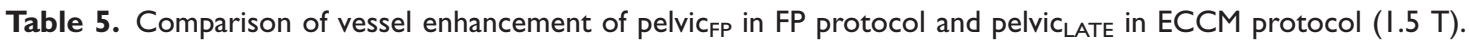

\begin{tabular}{|c|c|c|c|c|c|}
\hline & \multicolumn{2}{|c|}{ FP protocol } & \multicolumn{2}{|c|}{ ECCM protocol } & \multirow{3}{*}{$\frac{\text { Significance }}{P \text { value }}$} \\
\hline & \multicolumn{2}{|c|}{ Pelvic $_{\mathrm{FP}}(\mathrm{n}=14)$} & \multicolumn{2}{|c|}{ Pelvic $_{\text {LATE }}(n=14)$} & \\
\hline & Relative SI & SD & Relative SI & SD & \\
\hline Iliac veins & 88.73 & 32.33 & 61.10 & 37.87 & 0.04 \\
\hline IVC & 77.88 & 34.16 & 52.75 & 26.39 & 0.01 \\
\hline Aorta & 136.04 & 57.54 & 70.54 & 43.95 & 0.003 \\
\hline
\end{tabular}

ECCM, extracellular contrast medium; FP, first-pass; IVC, inferior vena cava; SI, signal intensity; SD, standard deviation.

(a)

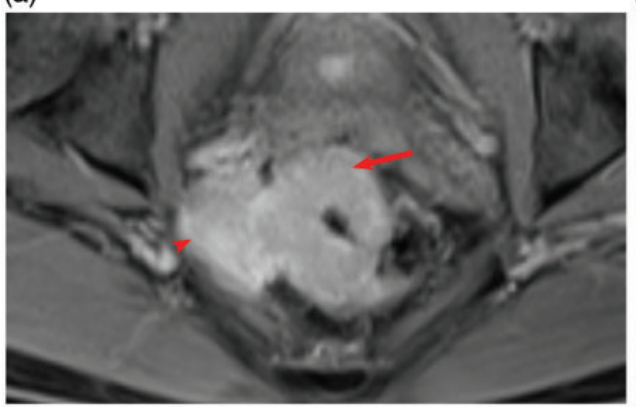

(c)

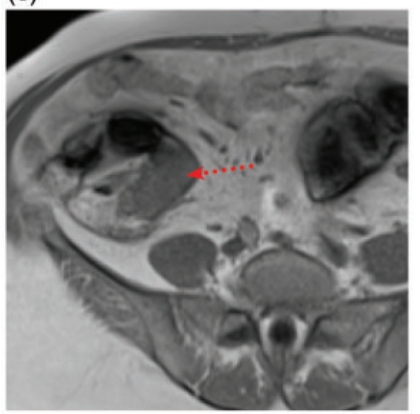

(b)

(d)

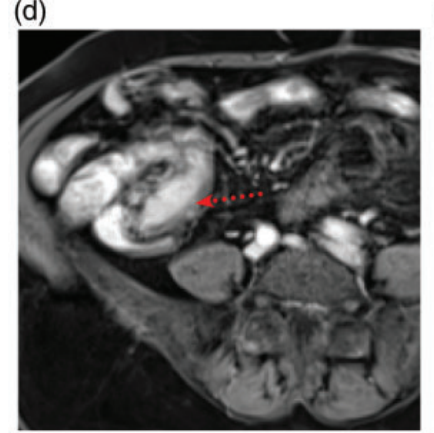

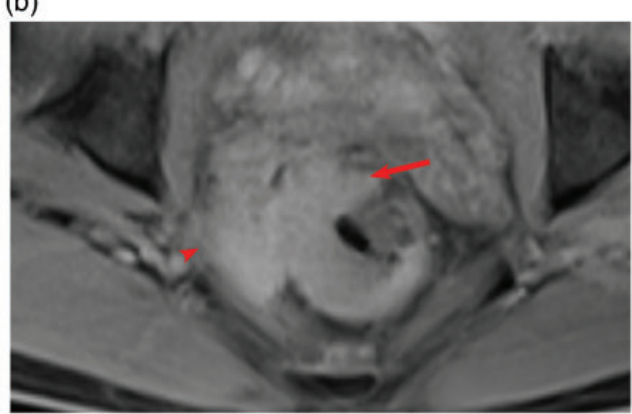

(e)

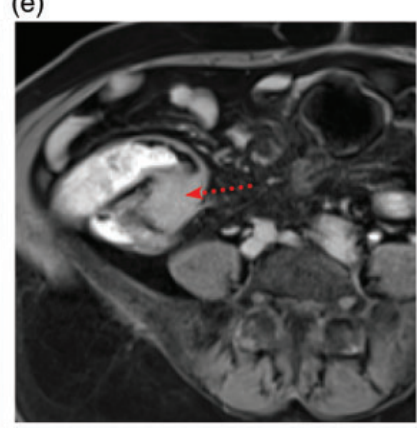

Fig. 3. Top: a 56 year-old male patient with an NEN of the rectum. The patient presents with a semi-circular, hypervascularized rectal tumor (arrow) and adjacent lymph node metastasis (arrowhead). Hypervascularity of the tumor is only visualized in pelvic $\mathrm{FP}_{\mathrm{F}}$ (a) pelvic $_{F P}$; (b) pelvic LATE. Bottom: a 62-year-old female patient with metastatic NEN of the terminal ileum with involvement of Bauhin's valve (dotted arrow). Hypervascularity is only visualized in pelvic $\mathrm{FP}_{\mathrm{F}}$ and shows the extended intraluminal tumor growth: (c) noncontrast TIW TSE; (d) pelvic FP; (e) pelvic LATE.

in the perfused compartment. This results in a lower extrahepatic parenchymal contrast enhancement in Gd-EOB MRI than in ECCM MRI in the upper abdomen $(10-12,14)$. Nevertheless, extrahepatic evaluation of the upper abdominal organs, especially of the pancreas and lymph nodes, is routinely performed in Gd-EOB MRI of the liver and good results regarding detection and differentiation of, for example, pancreatic neoplasms including neuroendocrine tumors, have been published $(15,16)$. It has also been shown that GdEOB MRI is adequate in extrahepatic lesion detection in rectal carcinoma patients (17). This holds true for the level of the liver, which is included in the regular dynamic first-pass hepatic contrast phases comprising the entire upper abdomen. The lower abdomen, however, even in scanners with a large transaxial field of view (FOV; in the range of $40-45 \mathrm{~cm}$ along the z-axis), has to be scanned at a different table position to facilitate homogeneous images in patients that are approximately $>150 \mathrm{~cm}$. This problem aggravates in modern scanners with wider and shorter gantry, like used in the present study (Siemens Aera and Skyra: width $=70 \mathrm{~cm}$; length $=60 \mathrm{~cm}$; FOV along $\mathrm{z}$-axis $=35 \mathrm{~cm}$ approximately).

With conventional interpolated $3 \mathrm{D}$ T1W breathhold sequences, the lower abdomen would only be examinable after the dynamic scans of the liver region as optimal liver imaging with Gd-EOB does of course 
(a)

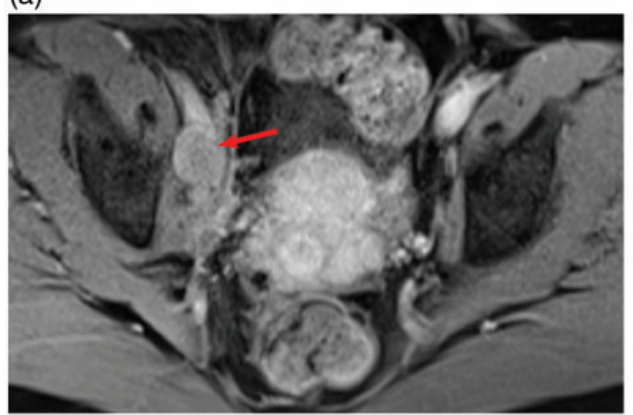

(c)

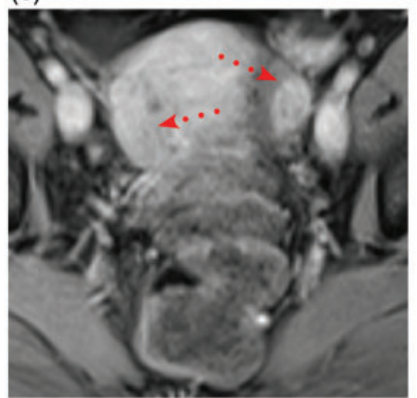

(b)

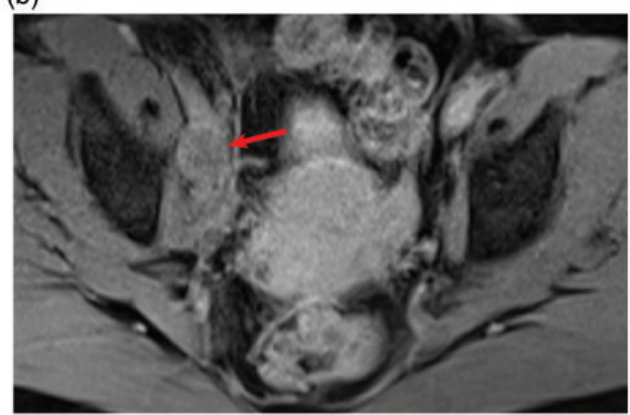

(e)
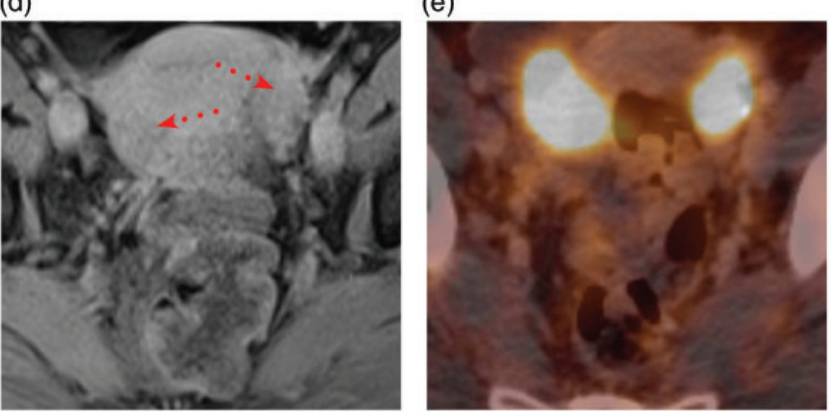

Fig. 4. Top: a 64-year-old female patient with uterine leiomyomas and pelvic lymph node metastases (arrow) of an NEN of the cecum:

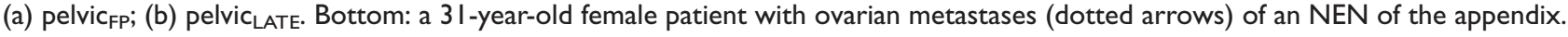
In pelvic $F_{F P}$, the tumor masses are definable from adjacent structures in comparison to pelvic LATE: (c) pelvic $_{F P}$; (d) pelvic LATE$_{\text {; }}$ (e) FDGPET-CT.

include all dynamic contrast phases during the first vascular pass (i.e. arterial, portal venous, venous, transitional/equilibrium phases). Including a subsequent extra sequence of the lower abdomen just after the completion of dynamic liver scanning would result in a delay of approximately $3 \mathrm{~min}$ from the beginning of the contrast injection as recorded in our study or even more with slower 3D T1W sequence technology. In this time period, recirculation of the entire contrast bolus already takes place and the administered Gd-EOB is diluted by distribution throughout the whole body and rapidly leaves the blood pool within a few minutes (18). During this time span, Gd-EOB is also extracted from the blood pool by the kidneys and also by the liver to a relevant amount. This results in poorer contrast enhancement of anatomical structures in such a late lower abdomen scan with Gd-EOB compared with a similar image acquisition protocol with ECCM. Therefore, this approach appears to be not equivalent to ECCM-enhanced MRI, the accepted gold standard in whole abdominal tumor staging, regarding extrahepatic diagnostic reliability. To overcome this shortcoming of Gd-EOB, we tried to insert an early scan of the lower abdomen and pelvis already during the vascular first pass of the contrast bolus. The purpose was to imitate with the pelvic $_{\mathrm{FP}}$ the conditions at the level of the liver during the dynamic contrast phases, where extrahepatic staging as discussed above was shown to be diagnostically equivalent to ECCM and GdBOPTA-enhanced MRI (10). With the multichannel parallel imaging technique, it is possible to scan the entire liver (approximately $22 \mathrm{~cm}$ along the z-axis) with the upper abdomen in $<10 \mathrm{~s}$ breath-hold technique without the need of increasing slice thickness to $>3-4 \mathrm{~mm}$. This enables table shuttling to center on the lower abdomen and pelvis and acquire 3D breath-hold imaging of this region still in an early vascular first-pass contrast phase at a delay of approximately $124 \mathrm{~s}$ as recorded in the present study.

The risk of insertion of a lower abdominal sequence during multi-phase liver evaluation is a delay of the subsequently acquired venous and transitional ("equilibrium") liver phase sequences, which was in the present study postponed by $54 \mathrm{~s}$ from the standard delay. This, however, did not lead to a significant difference of enhancement of hepatic structures. Only enhancement of the portal vein (in the venous phase) showed higher signal intensities in the standard protocol. However, diagnostic confidence should not be reduced by this result. Detection of portal vein pathologies as thrombosis or occlusion are preferably evaluated in the portal venous phase, which is not influenced by the pelvic ${ }_{F P}$ sequence. Acquisition of the pelvic $_{F P}$ sequence between the portal venous and venous/ transitional phases allows optimal timing of the venous and transitional phase with a delay of 
approximately $2-3 \min$ (152 s and $181 \mathrm{~s}$ in our study) in line with the recommendation of the consensus report of the International Forum for liver MRI (19).

An alternative to this approach would be to use a different liver-specific contrast agent with stronger vascular enhancement properties. Gd-BOPTA as the only alternative hepatocyte-specific contrast agent on the market shows longer extrahepatic vessel enhancement than Gd-EOB (18). However, because of the earlier (simplifying the workflow) hepatobiliary phase, Gd-EOB is often the preferred hepatocyte-specific contrast agent (14).

A minor limitation of the study is the slice thickness of $3 \mathrm{~mm}$ in dynamic liver evaluation. The detection of small high vascular liver metastases and multiplanar reconstructions could be impaired compared to a slice thickness of $1-2 \mathrm{~mm}$. However, this disadvantage is covered by a thin slice $(1 \mathrm{~mm}) 3 \mathrm{D} \mathrm{T} 1 \mathrm{~W}$ liver sequence (STARVIBE) in the hepatobiliary phase. As an important limitation of the study, diagnostic performance in terms of lesion detection was not evaluated. The study focused on technical feasibility and optimized imaging quality regarding quantified contrast enhancement of representative anatomical structures. Comparison of image noise and movement artifacts is not helpful as the sequence per se is already established in the clinical routine for contrast-enhanced MRI and was used for all parts of contrast imaging in the present study. Therefore, relevant differences in these parameters are not to be expected. Analyzing the detection rate in this heterogeneous patient group, even though a positive effect of the rather early contrast phase could be expected because of the tending high arterialization of NEN, would not be helpful as the results might be of limited transferability to other tumor entities $(20,21)$.

In turn, because of the general imaging enhancement aspect of our study, the results of the proposed pelvic ${ }_{\mathrm{FP}}$ shuttle protocol are not limited to the staging of NEN. The measured structures (aorta, IVC, iliac vessels, and lymph nodes) can be seen as representative markers with little inter-individual variation for ensuring the desired level of contrasting in the lower abdomen enabling a proper image interpretation.

In conclusion, the dilemma in whole abdominal staging of either choosing Gd-EOB for optimal hepatic lesion detection rate with the disadvantage of inferior performance in the lower abdomen and pelvis or administration of ECCM for optimal extrahepatic contrast enhancement but inferior accuracy inside the liver can be overcome by the shuttle protocol proposed herein (Fig. 1). The insertion of a first-pass contrast phase of the lower abdomen and pelvic region between the portal venous and venous liver phases with GdEOB is technically feasible using accelerated 3D interpolated breath-hold T1W sequence technology. The pelvic $_{\mathrm{FP}}$ sequence showed significant improvement of vessel and tissue contrast in the pelvis and lower abdomen at 1.5-T and 3-T MRI scanners compared to a commonly acquired late pelvic Gd-EOB sequence. There was no quality loss in hepatic enhancement compared to a standard Gd-EOB protocol. Gd-EOB pelvic $_{F P}$ vessel enhancement was not inferior to an equilibrium phase sequence with gadobutrol.

\section{Declaration of Conflicting Interests}

The author(s) of this manuscript declare relationships with the following companies: Bayer, Siemens.

\section{Funding}

The author(s) received no financial support for the research, authorship, and/or publication of this article.

\section{ORCID iD}

Uli Fehrenbach (D) http://orcid.org/0000-0003-3622-3268

Ute Fahlenkamp (D) http://orcid.org/0000-0002-0333-2962

\section{References}

1. Niederle B, Pape UF, Costa F, et al. ENETS consensus guidelines update for neuroendocrine neoplasms of the jejunum and ileum. Neuroendocrinology 2016;103:125-138.

2. Schmiegel W, Buchberger B, Follmann M, et al. S3Leitlinie - Kolorektales Karzinom. Zeitschrift fur Gastroenterologie 2017;55:1344-1498.

3. Huppertz A, Balzer T, Blakeborough A, et al. Improved detection of focal liver lesions at MR imaging: multicenter comparison of gadoxetic acid-enhanced MR images with intraoperative findings. Radiology 2004;230:266-275.

4. Grieser C, Steffen IG, Kramme IB, et al. Gadoxetic acid enhanced MRI for differentiation of FNH and HCA: a single centre experience. Eur Radiol 2014; 24:1339-1348.

5. Grieser C, Steffen IG, Seehofer D, et al. Histopathologically comfirmed focal nodular hyperplasia of the liver: gadoxetic acid-enhanced MRI characteristics. Magn Reson Imaging 2013; 31: 755-760.

6. Zech CJ, Korpraphong P, Huppertz A, et al. Randomized multicentre trial of gadoxetic acidenhanced MRI versus conventional MRI or CT in the staging of colorectal cancer liver metastases. Br J Surg 2014;101:613-621.

7. Bluemke DA, Sahani D, Amendola M, et al. Efficacy and safety of MR imaging with liver-specific contrast agent: U.S. multicenter phase III study. Radiology 2005;237:89-98.

8. Kahn J, Posch H, Steffen IG, et al. Is there long-term signal intensity increase in the central nervous system on T1-weighted Images after MR imaging with the hepatospecific contrast agent gadocetix acid? A cross-sectional study in 91 patients. Radiology 2017; 282: 708-716.

9. FDA. FDA Drug Safety Communication: FDA warns that gadolinium-based contrast agents (GBCAs) are 
retained in the body; requires new class warnings. Washington, DC: FDA, 2017.

10. Kim MJ, Kim SH, Kim HJ, et al. Enhancement of liver and pancreas on late hepatic arterial phase imaging: quantitative comparison among multiple gadolinium-based contrast agents at 1.5 Tesla MRI. J Magn Reson Imaging 2013;38:102-108.

11. Tamada $\mathrm{T}$, Ito $\mathrm{K}$, Sone $\mathrm{T}$, et al. Dynamic contrastenhanced magnetic resonance imaging of abdominal solid organ and major vessel: comparison of enhancement effect between Gd-EOB-DTPA and Gd-DTPA. J Magn Reson Imaging 2009;29:636-640.

12. Ringe KI, Boll DT, Husarik DB, et al. Lesion detection and assessment of extrahepatic findings in abdominal MRI using hepatocyte specific contrast agents-comparison of Gd-EOB-DTPA and Gd-BOPTA. BMC Med Imaging 2013;13:10.

13. Kirshbom PM, Kherani AR, Onaitis MW, et al. Carcinoids of unknown origin: comparative analysis with foregut, midgut, and hindgut carcinoids. Surgery 1998;124:1063-1070.

14. Feuerlein S, Gupta RT, Boll DT, et al. Hepatocellular MR contrast agents: enhancement characteristics of liver parenchyma and portal vein after administration of gadoxetic acid in comparison to gadobenate dimeglumine. Eur J Radiol 2012;81:2037-2041.
15. Kuhn JP, Hegenscheid K, Siegmund W, et al. Normal dynamic MRI enhancement patterns of the upper abdominal organs: gadoxetic acid compared with gadobutrol. Am J Roentgenol 2009;193:1318-1323.

16. Jang KM, Kim SH, Lee SJ, et al. The value of gadoxetic acid-enhanced and diffusion-weighted MRI for prediction of grading of pancreatic neuroendocrine tumors. Acta Radiol 2014;55:140-148.

17. Huppertz A, Franiel T, Wagner M, et al. Whole-body MRI with assessment of hepatic and extraabdominal enhancement after administration of gadoxetic acid for staging of rectal carcinoma. Acta Radiol 2010;51:842-850.

18. Brismar TB, Dahlstrom N, Edsborg N, et al. Liver vessel enhancement by Gd-BOPTA and Gd-EOB-DTPA: a comparison in healthy volunteers. Acta Radiol 2009;50:709-715.

19. Tanimoto A, Lee JM, Murakami T, et al. Consensus report of the 2nd International Forum for Liver MRI. Eur Radiol 2009;19(Suppl. 5):S975-989.

20. Ruf J, Schiefer J, Furth C, et al. 68Ga-DOTATOC PET/ CT of neuroendocrine tumors: spotlight on the CT phases of a triple-phase protocol. J Nucl Med 2011;52:697-704.

21. Baur AD, Pavel M, Prasad V, et al. Diagnostic imaging of pancreatic neuroendocrine neoplasms ( $\mathrm{pNEN}$ ): tumor detection, staging, prognosis and response to treatment. Acta Radiol 2016;57:260-270. 